\title{
Evaluate the Effectiveness of ECT in Borderline Personality Disorder Resistant To Treatment
}

\author{
Mohammad hasan Baniasad ${ }^{1}$, Zahra Zolfaghari ${ }^{2}$, Hosein payandan ${ }^{3}$, \\ Nasim Sadeghi ${ }^{4}$, Jaber Davoodi ${ }^{5}$, Seyedeh Samera Hoseini ${ }^{6}$
}

\section{ABSTRACT}

Aim: It's different fields of borderline personality disorder and is associated with characteristics of turbulence and emotions of anxiety, anger, depression and risky behaviors such as self-injury and drug abuse. Hospitalization for ECT provides an opportunity to begin treatment, change of previous therapy or management of the crisis. Electroconvulsive therapy is the most effective psychiatric treatment that in this study investigated its effectiveness in patients with borderline personality disorder resistant to treatment. Method: The method of this study was Case Series which were selected by available sampling, samples of 10 patients with borderline personality disorder were resistant to treatment and were assessed by using of check list Frequency of behavior- researcher made-. Results: The finding yielded that criteria of sadness, anxiety and restlessness and feeling of absurdity, in evaluation was performed by families to examine the possible changes, after ECT therapy decreased compared to baseline. As well as after ECT therapy verbal and nonverbal expression of affection criterion represents an increase compared to baseline. Conclusions: According to the findings Electroconvulsive therapy can affect on mood symptoms associated in patients borderline.

Keywords: Electroconvulsive Therapy, Borderline Personality Disorder, Resistant To Treatment

Borderline personality disorder is the most common personality disorder in psychiatric settings ${ }^{1}$ and today is one of ten classification of personality disorder ${ }^{2}$. The prevalence of BPD in the general population is about one percent. Prevalence rate of this disorder in the general population

\footnotetext{
${ }^{1}$ Assistant Professor at Islamic Azad University, Tehran

${ }^{2}$ Assistant Professor at Islamic Azad University, Tehran

${ }^{3}$ Psychometry, Shahid Doctor Lavassani Hospital, Tehran

${ }^{4} \mathrm{MD}$, Semnan, Iran

${ }^{5}$ M.A in Psychology of Family Therapy, Family Reaserch institute of Shahid Beheshsi University, Tehran, Iran

${ }^{6}$ Student of Ph.D in Psychology, Semnan University, Semnan, Iran

*Responding Author

(C) 2016, H Baniasad, Z Zolfaghari, H Payandan, N Sadeghi, J Davoodi, S Hoseini; licensee IJIP. This is an Open Access Research distributed under the terms of the Creative Commons Attribution License (http://creativecommons.org/licenses/by/2.0), which permits unrestricted use, distribution, and reproduction in any Medium, provided the original work is properly cited.
} 


\section{Evaluate the Effectiveness of ECT in Borderline Personality Disorder Resistant To Treatment}

is approximately $2 \%$, and is estimated in the outpatient mental health clinics, approximately $10 \%$ and approximately $20 \%$ of patients in psychiatric wards. The scope of this disorder in the clinical populations suffering from personality disorders from is $30 \%$ to $60 \%$ and is more common in women ${ }^{3}{ }^{4}$. According to DSM $-5^{7}$, this disorder is characterized as "a pervasive pattern of instability in interpersonal relationships, self-image, mood impulsivity that begins in early adulthood and emerges in different situations and environments" ${ }^{3}$. Rul, Useda, Confort and Doan found that borderline personality disorder is associated with patterns of problematic mood, including uncontrollable anger and emotional instability ${ }^{5}$. According to one study, $6.1 \%$ of adults in the United States of America during a year of study had borderline personality disorder ${ }^{4}, 6,7$.

Most therapists who have faced in the clinical setting with patients with borderline personality disorder, agree with the fact that the treatment of these patients is difficult ${ }^{5}, 6,8$. Characteristics of borderline in old Psycho Analytic article and by individuals like Moore, Reich, Oberndorf, Glover, Kasanin and Fine observed and discussed ${ }^{9}$, however the term "borderline" was used first time by Stern in 1938. Stern used this term to describe people who were on the border between neurotic and psychotic states; This means that patients who are under intense psychological pressure are affected to psychotic behaviors and thought processes and quickly returned to normal levels of mental function ${ }^{10}$. Currently extensive epidemiologic research about BPD show that the clinical course of this disorder is not disappointing, as it was during the 1940s until the early 1990s ${ }^{11}$. However, the rate of abandonment of treatment in them are very high and the improvement is variable ${ }^{12}$ and only a few of them respond to psychiatric drug treatment, education of interpersonal relationships and control critical behaviors ${ }^{13}$. Most studies have focused on such treatment and have less attention on electroconvulsive therapy in treatmentresistant patients.

ECT is one of the oldest treatments that have been used in the case of mental illness that its history returns to sixteenth century ${ }^{14}$. This method is used in the cases of patient does not respond to any drug treatment or can not tolerate drug side effects or severe symptoms of psychosis and desire to commit suicide or homicide and the issues that need immediate treatment response $1^{15}, 1^{6}$. Also, it looks like a minor surgical procedure that requires a series of preparatory and primary health care ${ }^{17}$. ECT is the electrical stimulation of the brain planned for the beginning of the seizure ${ }^{18}$. Electrical stimulation resulted in a general tonic activity for about ten seconds and then continues to the general clonic activity for variable time (from several seconds to more than a minute). There is a general consensus on the fact that the duration of the seizure is a determining factor in the efficacy of ECT, but the general belief is changing recently $1^{9}, 2^{\circ}$. Because the $80 \%$ of patients with borderline personality disorder have behaviors and suicide attempts, and finally $4 \%$ to $9 \%$ of them die by suicide, and this is almost 50 times higher than the

${ }^{7}$ Fifth edition of the Diagnostic and Statistical Manual of Mental Disorders

(c) The International Journal of Indian Psychology, ISSN 2348-5396 (e)| ISSN: 2349-3429 (p) | 39 


\section{Evaluate the Effectiveness of ECT in Borderline Personality Disorder Resistant To Treatment}

mortality rate for suicide in the general population ${ }^{21,22}$ as well as other unsuccessful attempts and self-harm of these patients that often leads to maim, brings a huge burden on the healthcare system and family. Therefore put this disease one of the greatest challenges in psychiatry. Also if symptoms of personality disorder persisted a long time, leads to attention deficit in the performance of individual, social, employment and education. These, along with impulsive behavior, instability in relationships and emotional, with rapid changes in mood $23,2^{4}$ and in case of relapse and resistance to treatment routine, to prevent damage to the individual and society need to serious intervention. So we need to deal with the present study examines the effectiveness of ECT for such severe cases of borderline personality disorder.

\section{METHOD}

\section{Instruments}

The instrument used in this study include the frequency of behavior checklist, made by the researcher and two-part that was based on the literature review. The first part of the frequency behavior of the checklist, is related to demographic (age, gender, marital status). In the second part 29 questions related to the recording of the frequency behavior, that frequency of behaviors recorded during a month by the family. Its validity was approved by two faculty members. Its reliability was confirmed by Cronbach's alpha coefficient $(\alpha=0 / 85)$ and after completion of the pilot 20 checklist by families.

\section{Participants:}

This study is the type of observational studies, case reports and retrospective which was conducted between 2012 to 2013 years in Section of psychiatric Lavasani Hospital at Tehran. The study population were patients with diagnosis of borderline personality disorder that due to frequent relapses and hospitalization and lack of response to previous treatment, inability of their families in order to control them and have frequent conflicts with the legal system, have been diagnosed as resistant to treatment. Fifteen patients were selected for the sample group through available sampling. Ten patients up to full completion of the project was necessary and sufficient cooperation and five patients were excluded from the study for various reasons, including lack of cooperation with the health system and lack of regular follow up.

\section{Procedure:}

For one month before the start of ECT, the families of the patients were trained to observe and record the behavior of the subjects and the frequency behavior in frequency of behavior checklist. As well as non-visible symptoms were recorded by interviewing the patients and their families at the end of this month. This frequency were considered as the baseline. In the next step patients hospitalized in the psychiatric ward and received ECT, in addition to other treatments. Considering the condition of ethical research, after obtaining informed consent from families and do the necessary assessments before electroconvulsive, including examination of complete physical and neurological checkups, medical history of the patient, observation before 


\section{Evaluate the Effectiveness of ECT in Borderline Personality Disorder Resistant To Treatment}

anesthesia, performing laboratory evaluations include $\mathrm{CBC}$ diff, urine analysis, chest radiography, electrocardiography and after discontinuation of drugs that interfere with ECT, was used bilateral ECT under general anesthesia.

After the ECT all patients were ventilated with 100\% oxygen until the complete return of spontaneous breathing. During ECT patients were followed every week and after the achieve maximum therapeutic response (The order of maximum therapeutic response is after 2 consecutive ECT patient does not feel more improvement.) with conservative drug therapy that was similar in all of them, were discharged. During six months patients repeatedly experienced recurrence of symptoms which sometimes leads to hospitalization in psychiatric wards. But all of the patients were hospitalized only twice, with receiving ECT that during the twice hospitalized they received 24 sessions of ECT (every time during the one-month hospitalization, received 12 sessions ECT.) with the conditions described in addition to conventional therapy.

Since in this study, ECT were considered as experimental variables and change of signs at the end of six months assessed using the frequency of behavior checklist, therefore again asked from families of patients for a month to observe their patients signs and record the frequency of signs in frequency of checklist. Because it was necessary have passed at least one month since the last electroconvulsive to temporary Amnesia and cognitive impairment which is a common side effect of ECT, does not distort the results. Then again patients for six months were followed for recurrence and its relation to therapeutic response that this frequencies were considered as the second baseline (Research design ABA). After collecting data by using SPSS software were analyzed. Also, because the previous treatments for these patients was unsuccessful, can be considered the changes observed in the subsequent behaviors compared to baseline due to experimental variables (ECT).

\section{RESULTS}

Demographic information (such as age and history of medications and underlying disease) was extracted from patients' hospital files. Information about the apparent Seizure (observed tonic and clonic contractions by psychiatrist) and the actual seizure (study ECG strip in terms of spike \& sharp waves by psychiatrist) and hemodynamic variables by using Pulse Oximetry monitoring, non-invasive measurement of blood pressure and ECG monitoring by anesthesia technicians were collected and recorded in relevant forms. According to information obtained; Participants in the study were aged between 49-19 years and the mean age of them was 32 years old. In addition 6 patients (60\%) were married, 4 patients (40 percent) were single. For entering the study, were considered criteria for subjects which including the following:

1- According to DSM-5 be diagnosed with borderline personality disorder that are approved by a psychiatrist. 2- Medications and other therapeutic interventions for patients is ineffective. 3Have indications for electroconvulsive therapy. 4- Have consent to participation in this research. 
The exclusion criteria consisted of; 1- Have a history of Cardiovascular disease. 2- Have a history of hypertension. 3- Suffering from Pheochromocytoma. 4- Have a history of drugs that affect on the duration of the seizure.

In this study data analysis method is descriptive. The results from checklist, related to before and after the experimental variable were compared with each other via charts. For each subject a graph was plotted and analyzed that in this graphs observed behaviors on the horizontal axis and the frequency related to them, was plotted on the vertical axis (Graphs no 1, 2, 3, 4).

Graph no 1: Comparison of frequency of criteria "sad" before and after ECT

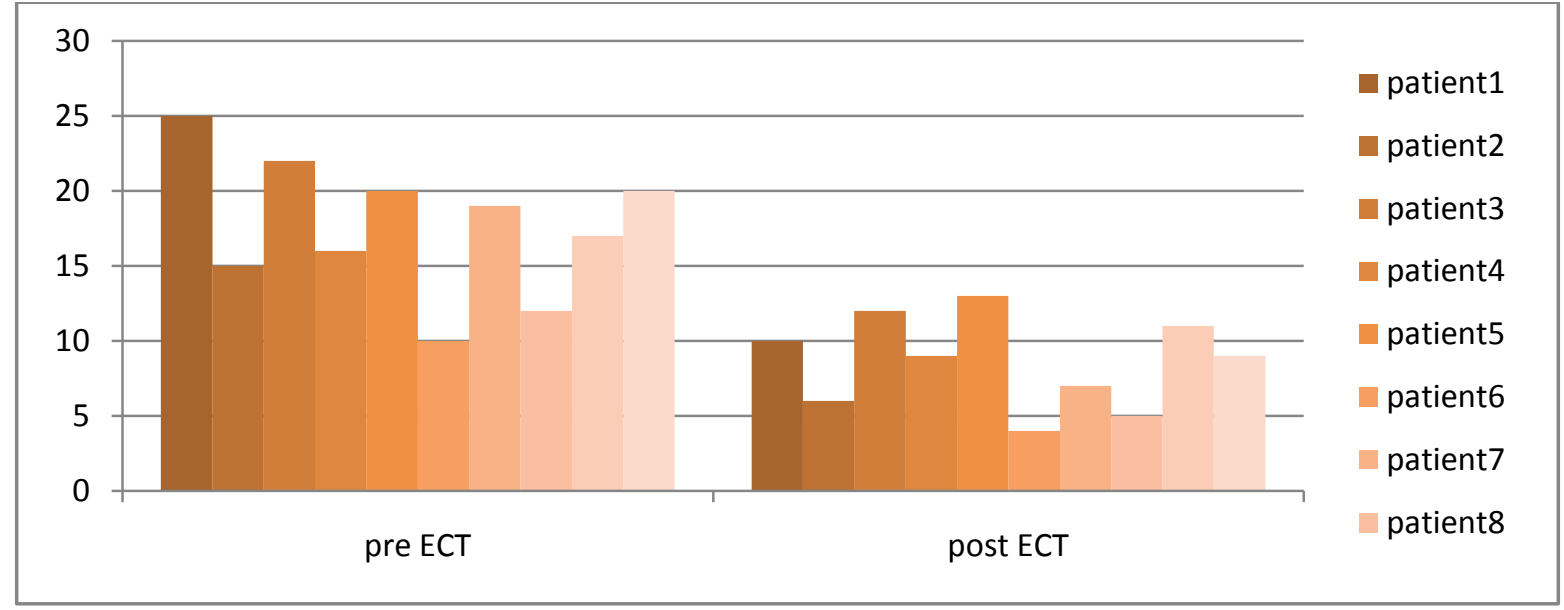

Graph no 2: Comparison of frequency of criteria "anxiety and restlessness" before and after ECT

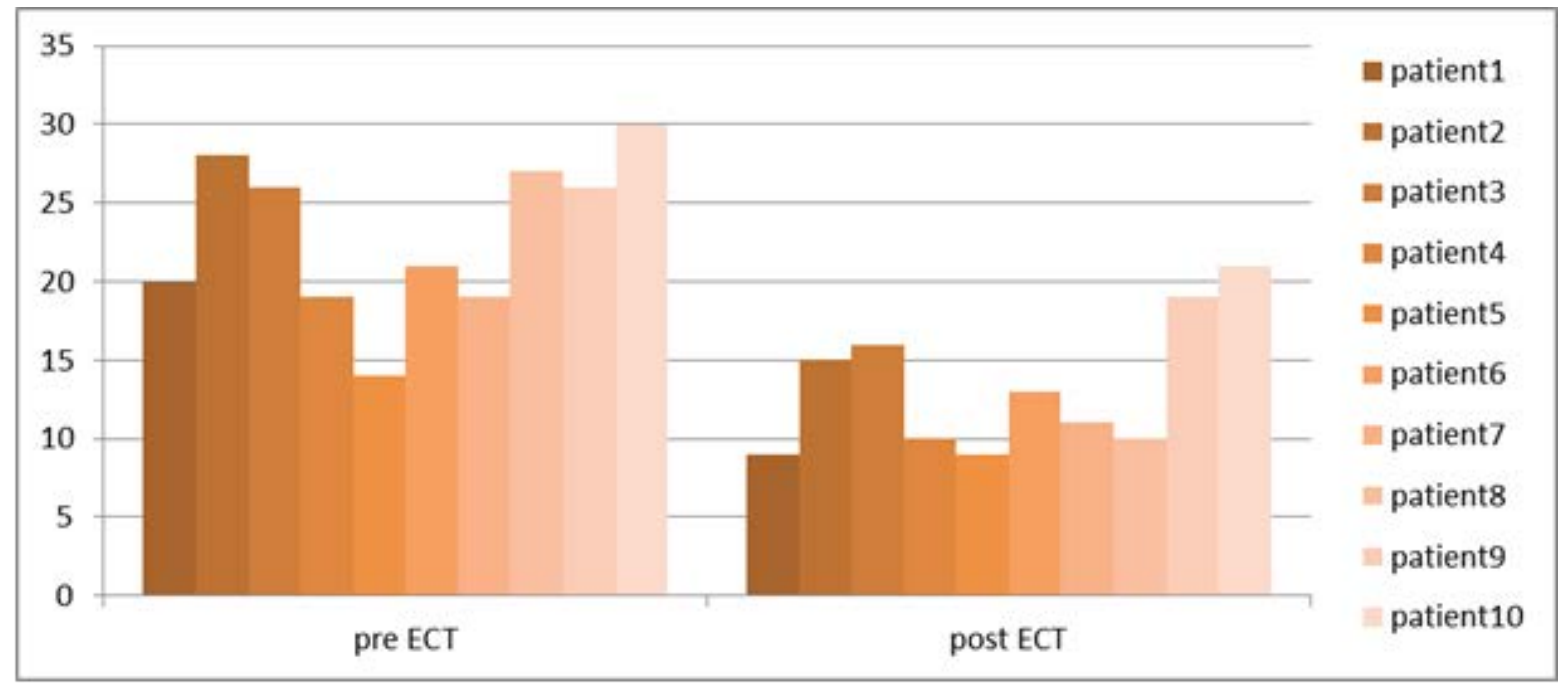


Graph 3: Comparison of frequency of criteria "feeling of emptiness" before and after ECT

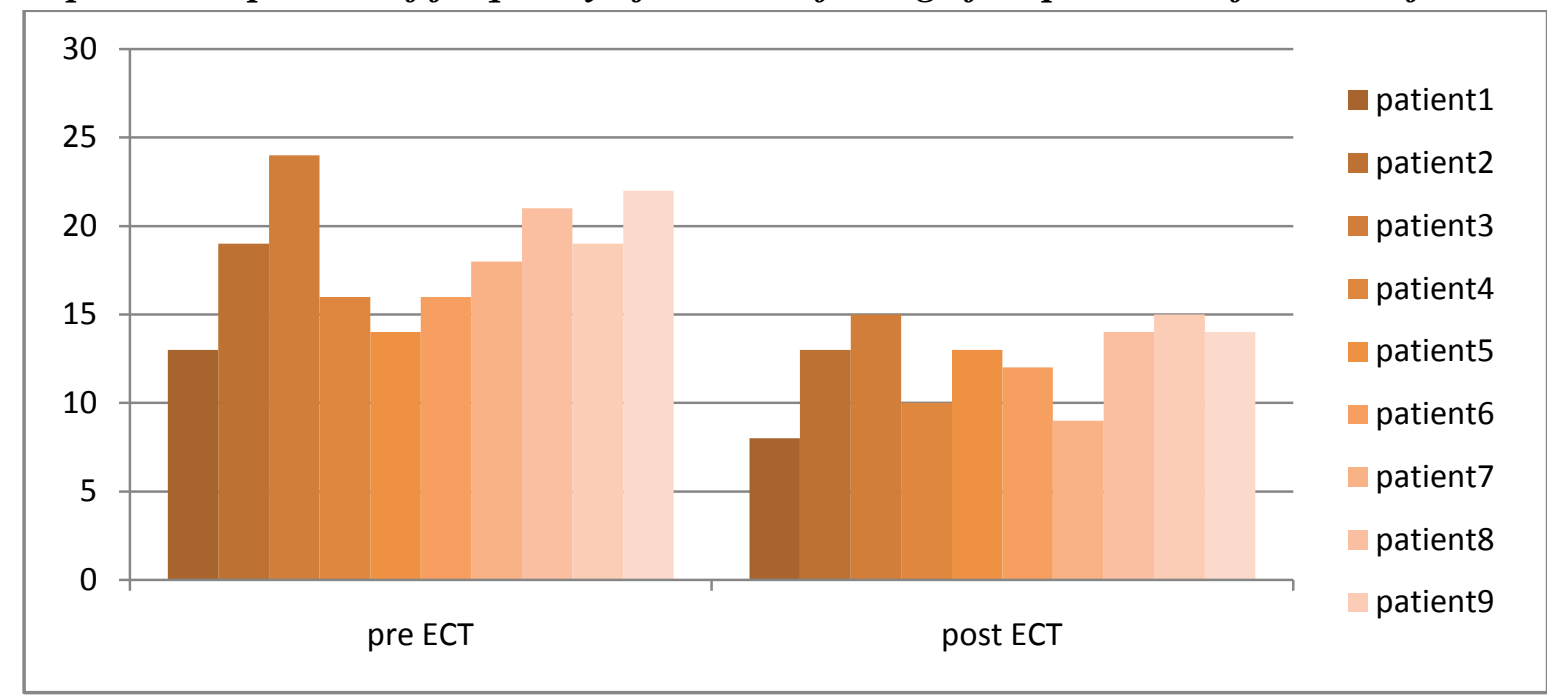

According to the behavior of the subjects in checklist, frequency of behaviors indicate significant decline. However, some behaviors showed a more significant decline. As specified in the above graphs, Frequency of criteria "sad", "anxiety and restlessness" and "feeling of emptiness", among behaviors in the checklist, in each of the ten subjects after ECT has decreased compared to baseline. In addition among behaviors in the checklist the criterion of "verbal and nonverbal affective expression" represents an increase compared to the baseline (Graphs no 4).

\section{Graph no 4: Comparison of frequency of criteria "verbal and nonverbal affective expression"} before and after ECT

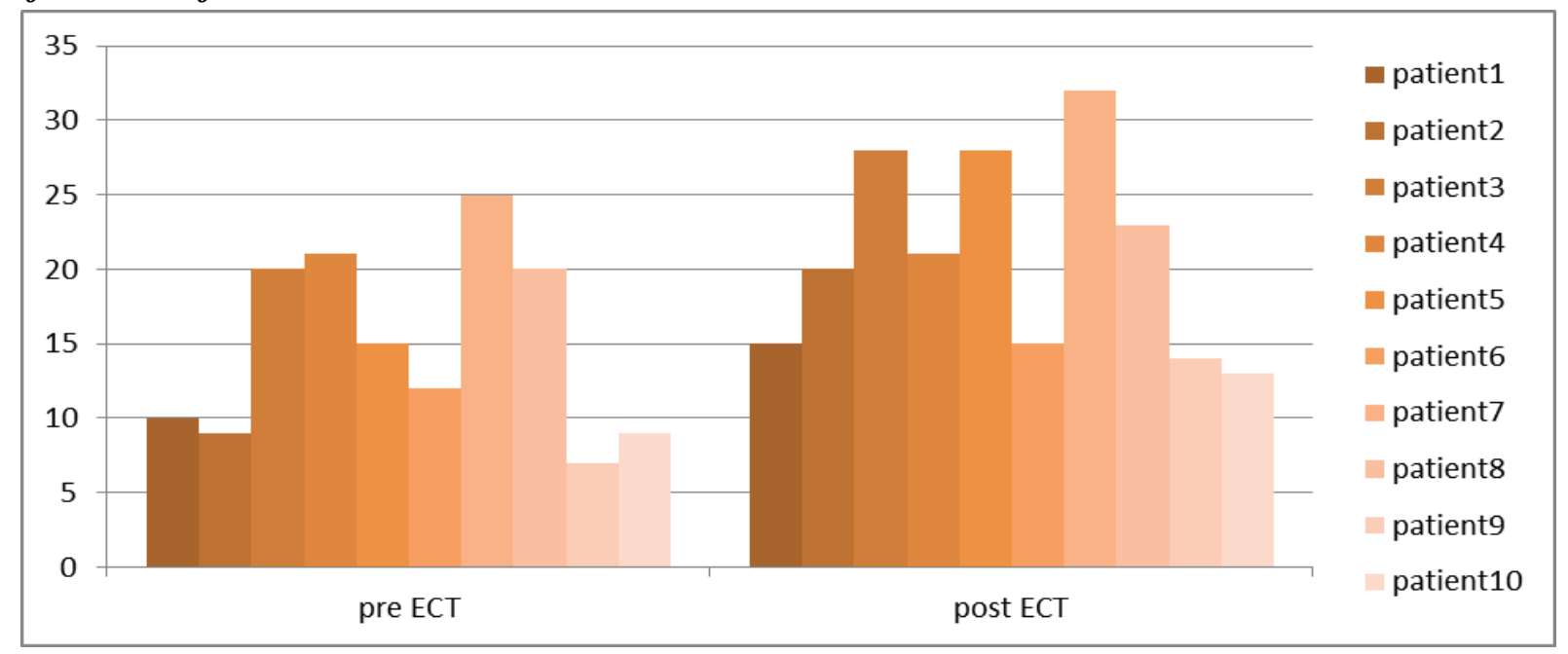

\section{CONCLUSION AND DISCUSSION}

The purpose of this study was evaluating the effectiveness of ECT in borderline personality disorder resistant to treatment. The results of this study showed that frequency of behavior in all criteria was significantly reduced; however, it is different amount of this reduction from one patient to another and from behavior to another behavior. But it is clear that all subjects showed

(C) The International Journal of Indian Psychology, ISSN 2348-5396 (e)| ISSN: 2349-3429 (p) | 43 


\section{Evaluate the Effectiveness of ECT in Borderline Personality Disorder Resistant To Treatment}

varying degrees of response to treatment. In all ten subjects criteria of sadness, anxiety and restlessness, feeling of emptiness among behaviors in the checklist after ECT was lower from baseline and this indicate electroconvulsive therapy was effective in improving mood symptoms and reduce anxiety. According to the results it can be said that electroconvulsive therapy, according to results of previous research decreases mood symptoms associated with depression and anxiety in people with borderline personality disorder ${ }^{25}, 2^{6}, 2^{7}$. To explain these findings can be noted to the following factors:

- Borderline personality disorder often associated with emotional states of anxiety, depression and risky behaviors such as self-harming and maximum overlap of this disorder is major depressive disorder, that is associated with symptoms of low mood, anxiety, sadness ${ }^{3}$.

- American Psychiatric Association based on the evidence obtained from research recommended ECT in patients with borderline personality disorder with severe and resistant to treatment ${ }^{24}$. Due to the overall reduction of symptoms, according to the criteria in research findings, confirm the fact that most decrease is related to criteria that they have more proximity with mood and depression signs and ECT has great effect on symptoms that are more or less observed in mood disorders ${ }^{25}$.

- Considering that ECT and its recovery in patients with borderline personality disorder is not just a statistical findings but also is more a clinical result, therefore this result would be consistent with views of those that consider borderline personality disorder a type of mood disorder ${ }^{25}$. Report published by the American Psychiatric Association suggests that the main purpose of using ECT in patients borderline is relieve symptoms in patients who have axis I disorders - Mood disorders- that is seen in more than half of the patients borderline hospitalized ${ }^{26}$.

However, inconsistent with findings that suggest ECT effect on along mood disorders in patients borderline $2^{5}, 2^{7}$ Farmer et al. (2006) claimed the simultaneous presence of borderline personality disorder and depression, reduces the response to ECT ${ }^{28}$.

- To explain this discrepancy can say that it seems that discrepancy relevant to methodological weaknesses, lack of standardized diagnostic criteria and lack of long-term follow-up.

Another finding of the present study was that the criteria "verbal and nonverbal affective expression" after ECT, increased compared to the baseline. To explain these findings can be noted to the following factors:

- In treatment with ECT if response to treatment is good short waves In the prefrontal cortex increases. Prefrontal cortex is located in front of the head that has an important role in controlling stimuli and emotional states. To find out such a response can be paid to the patient's observed behaviors, including verbal and nonverbal affective expression that increases looking to do ECT ${ }^{29}$. 


\section{Evaluate the Effectiveness of ECT in Borderline Personality Disorder Resistant To Treatment}

- Electroconvulsive by increasing the release of hormones from the hypothalamus and pituitary the impact on neurotransmitters and affect person's emotional states ${ }^{29}$.

Finally, with regard to the above issues and the goals of study was looking for, it can be said ECT can affect on mood symptoms in patients borderline. On the other hand since currently does not exist a study that examined the effects of ECT on patients suffering from personality disorders and personality traits, and the few studies have assessed personality (using personality assessment tests) before and immediately after completion of treatment with ECT sessions, believe that ECT clearly does not effect on personality traits ${ }^{18}$. But eventually a general look at the collection of symptoms and behavior, and changes can be considered as criteria for evaluation of the effectiveness of a therapeutic approach.

The limitations of this study; was small sample size and drop in the number of samples. In addition, because the study is a experimental design within the group and the sample is not representative of population, so there is no possibility of generalizing the results. Also for that further to realize to effectiveness of ECT in the treatment of patients with borderline personality disorder, long-term follow-up is recommended to be done about the outcome of treatment.

\section{Acknowledgments}

The author appreciates all those who participated in the study and helped to facilitate the research process.

\section{Conflict of Interests}

The author declared no conflict of interests.

\section{REFERENCES}

1 Durand, Vincent Mark, and David H. Barlow. Psychopathologie: une perspective multidimensionnelle. De Boeck Supérieur, 2002.

2 Stern, Adolph. "Borderline group of neuroses." The Psychoanalytic Quarterly (1938).

3 American Psychiatric Association. Diagnostic and Statistical Manual of Mental Disorders (DSM-5®). American Psychiatric Pub, 2013.

4 Torgersen, Svenn, Einar Kringlen, and Victoria Cramer. "The prevalence of personality disorders in a community sample." Archives of general psychiatry 58.6 (2001): 590-596.

5 Freeman, Arthur, Mark H. Stone, and Donna Martin. Comparative treatments for borderline personality disorder. Springer Publishing Company, 2005.

$6 \quad$ Oldham, John M. "Borderline personality disorder and suicidality." FOCUS (2013).

7 Lenzenweger, Mark F., et al. "DSM-IV personality disorders in the National Comorbidity Survey Replication." Biological psychiatry 62.6 (2007): 553-564.

8 Cloninger, C. Robert. "A practical way to diagnosis personality disorder: a proposal." Journal of Personality Disorders 14.2 (2000): 99-108. 


\section{Evaluate the Effectiveness of ECT in Borderline Personality Disorder Resistant To Treatment}

9 Gunderson, John G. Borderline personality disorder: A clinical guide. American Psychiatric Pub, 2009.

10 Paris, Joel, and Hallie Zweig-Frank. "A 27-year follow-up of patients with borderline personality disorder." Comprehensive psychiatry 42.6 (2001): 482-487.

11 Zanarini, Mary C., et al. "Time to attainment of recovery from borderline personality disorder and stability of recovery: A 10-year prospective follow-up study." American Journal of Psychiatry 167.6 (2010): 663-667.

12 Clarkin, John F. "Treatment of personality disorders." British Journal of Clinical Psychology 35.4 (1996): 641-642.

13 Soloff, Paul H. "Psychopharmacology of borderline personality disorder." Psychiatric Clinics of North America 23.1 (2000): 169-192.

14 Kaplan H, Sadok V. Comprehensive text book of psychiatry. 9th Ed. Philadephia: Lippincott Co; 2007; 375.

15 Scott, Allan IF. "College guidelines on electroconvulsive therapy: an update for prescribers." Advances in Psychiatric Treatment 11.2 (2005): 150-156.

16 Ghaziuddin, N., et al. "Work Group on Quality Issues; AACAP (2004) Practice parameter for use of electroconvulsive therapy with adolescents." J Am Acad Child Adolesc Psychiatry 43.12: 1521-1539.

17 Arkan, Burcu, and Besti Üstün. "Examination of the effect of education about electroconvulsive therapy on nursing practice and patient satisfaction." The journal of ECT 24.4 (2008): 254-259.

18 Sanacora, Gerard, et al. "Increased cortical GABA concentrations in depressed patients receiving ECT." (2014).

19 Lalla, Fiore R., and Thomas Milroy. "The current status of seizure duration in the practice of electroconvulsive therapy." Canadian journal of psychiatry 41.5 (1996): 299-304.

20 Sackeim, Harold A., et al. "Effects of stimulus intensity and electrode placement on the efficacy and cognitive effects of electroconvulsive therapy." New England Journal of Medicine 328.12 (1993): 839-846.

21 Zanarini, Mary C., et al. "The McLean Study of Adult Development (MSAD): overview and implications of the first six years of prospective follow-up." Journal of personality disorders 19.5 (2005): 505-523.

Trull, Timothy J., et al. "Borderline personality disorder features in nonclinical young adults: 2. Two-year outcome." Journal of Abnormal Psychology 106.2 (1997): 307.

Linehan, Marsha M., Bryan N. Cochran, and Constance A. Kehrer. "Dialectical behavior therapy for borderline personality disorder." Clinical handbook of psychological disorders: A step-by-step treatment manual 4 (2001): 365-420.

24 American Psychiatric Association, and American Psychiatric Association. Work Group on Borderline Personality Disorder. Practice guideline for the treatment of patients with borderline personality disorder. American Psychiatric Pub, 2001. 


\section{Evaluate the Effectiveness of ECT in Borderline Personality Disorder Resistant To Treatment}

25 Farrell, Joan M., Ida A. Shaw, and Michael A. Webber. "A schema-focused approach to group psychotherapy for outpatients with borderline personality disorder: a randomized controlled trial." Journal of Behavior Therapy and Experimental Psychiatry 40.2 (2009): 317-328.

26 Davidson, Richard J., Daren C. Jackson, and Ned H. Kalin. "Emotion, plasticity, context, and regulation: perspectives from affective neuroscience." Psychological bulletin 126.6 (2000): 890.

27 Binks, C. A., et al. "Psychological therapies for people with borderline personality disorder." Cochrane Database Syst Rev 1 (2006): Art.

28 Farmer, Richard F., and Rosmery O. Nelson-Gray. Personality-guided behavior therapy. American Psychological Association, 2005.

29 Blais, Mark A., et al. "Stability and predictive value of self-report personality traits pre-and post-electroconvulsive therapy: a preliminary study." Comprehensive psychiatry 39.4 (1998): 231-235.

How to cite this article: H Baniasad, Z Zolfaghari, H Payandan, N Sadeghi, J Davoodi, S Hoseini (2016), Evaluate the Effectiveness of ECT in Borderline Personality Disorder Resistant To Treatment, International Journal of Indian Psychology, Volume 3, Issue 4, No. 65, ISSN 2348-5396 (e), ISSN: 2349-3429 (p), DIP: 18.01.138/20160304, ISBN: 978-1-365-34680-4 